\title{
LA ENSEÑANZA DE LA TRADUCCIÓN COMERCIAL (INGLÉS-ESPAÑOL): UNA PROPUESTA FORMATIVA
}

\author{
Karina Socorro Trujillo \\ Karina.socorro@ulpgc.es \\ Universidad de Las Palmas de Gran Canaria (ULPGC)
}

\section{Resumen}

Conciliar la traducción profesional del ámbito del Derecho Mercantil Internacional con la pedagogía es el primer objetivo que nos planteamos para crear un espacio de trabajo que favorezca la enseñanza de la traducción comercial con el fin particular de que el futuro egresado en traducción llegue a traducir con garantía de éxito y a resultar atractivo al empleador de este ámbito de especialidad. Partiendo de esta premisa, en este trabajo establecemos una propuesta metodológica que pone el foco en la fase preparatoria del aprendizaje, que, a nuestro modo de ver, contempla la comprensión del texto, la instrucción, la inmersión temática y, en definitiva, la adquisición de la competencia traductora del aprendiz de traductor, fundamental para desenvolverse en el mundo de la traducción comercial profesionalmente.

\begin{abstract}
"Teaching commercial translation (English-Spanish): A teaching proposal"

Reconciling professional International Trade Law with translation teaching is the first aim that we pursue in this paper. Our ultimate objective is to create a working space appropriate for teaching translation for commercial purposes. Thus, we believe that future translation graduates will be able to translate successfully since they will develop skills that will make them more appealing to employers in this area. Under this assumption, we present a methodological proposal focused on the preparatory stage which, in our opinion, favors understanding, translation training as well as thematic immersion. On the whole, we aim to develop full translation competence among translation trainees enabling them to translate professionally for commercial purposes.
\end{abstract}


Palabras clave: Traducción comercial. Competencias. Tipología textual. Convenciones textuales. Glosarios.

Keywords: Commercial translation. Competences. Textual typology. Text conventions. Glossaries.

Manuscript received on June 30, 2015 and accepted for publication on October 29, 2015. 


\section{Introducción}

Como sabemos, uno de los elementos clave del Espacio Europeo de Educación Superior (EEES) es que las universidades deben mantener la responsabilidad de formar profesionales capaces y competentes en los ámbitos laborales específicos relacionados con cada titulación, y, por tanto, es preciso partir del perfil profesional a la hora de diseñar un programa de estudios. Según el Fondo Monetario Internacional (FMI 2001: 1):

En las últimas décadas la economía mundial ha experimentado un rápido crecimiento, al que contribuye entre otros factores la aceleración del comercio internacional, fruto del avance tecnológico y de un esfuerzo concertado para reducir las barreras comerciales.

A la vista de la importancia que, en consecuencia, adquiere la traducción comercial, esta se imparte como materia en grados y postgrados de Traducción e Interpretación desde su creación en 2009. Así, en este trabajo, podrá comprobarse que contemplamos la docencia de la traducción comercial desde una perspectiva profesional. Para ello, entre otras cuestiones, abordamos la necesidad de que tanto el docente como el estudiante, desde el principio, conozcan las salidas y las necesidades profesionales. Pero, ¿qué se entiende por traducción comercial?:

La traducción comercial es la actividad que podemos encontrar bajo denominaciones diversas: traducción mercantil, para el comercio exterior, de comercio internacional y se asimila con la traducción jurídica a través del derecho mercantil, aunque no todos los textos que genera el comercio se pueden clasificar estrictamente como textos con valor jurídico (Mayoral 2006: 1).

[...] [T] ampoco resulta difícil encontrar elementos de coincidencia entre la economía, el comercio, las finanzas y la economía de la empresa pero también nos parece incontestable que cada una de estas actividades presenta a su vez rasgos particulares bien definidos (Mayoral 2006: 1-2).

Precisamente, debido a la novedosa y reciente incorporación de esta materia en los planes de estudio de Traducción e Interpretación -antes podía impartirse dentro de la asignatura de traducción económico-jurídica y a criterio del docente-, la investigación en torno a la traducción comercial tuvo sus inicios a finales del siglo XX. En España, destacan autores como Mayoral 
(2006, 2007, 2013), Suau Jiménez (2010), Román Mínguez (2012) y Medina Reguera $(2007,2009)$-dedicada a la combinación lingüística español-alemán-, Socorro Trujillo (2008, 2012) y Del Pozo Triviño (2009, 2014). Estas dos últimas autoras, además, han realizado trabajos monográficos centrados específicamente en los géneros del comercio internacional y en los documentos marítimos del ámbito legal, respectivamente. Por otra parte, hay que señalar que también en esta parcela de estudio se han desarrollado iniciativas materializadas en proyectos de investigación, como el de COMENEGO, un corpus multilingüe especializado en el ámbito de la economía y los negocios, útil como banco textual y favorecedor de la normalización terminológica del ámbito (Gallego 2013; Gallego \& Krishnamurthy 2013; Rodríguez-Inés 2014), o la celebración en Alicante en 2014 del Primer Congreso Internacional de Traducción Económica, Comercial, Financiera e Institucional, que logró reunir a profesionales, investigadores, profesores y demás colectivos interesados por la traducción de estos ámbitos de especialidad.

El Libro blanco del Título de Grado de Traducción e Interpretación recoge que el objetivo principal que se plantea la enseñanza de la traducción comercial es conseguir que el egresado disponga de los conocimientos y habilidades que le permitan asumir encargos de traducción de textos comerciales. Con este fin, se le han asignado las siguientes competencias: a) reconocimiento de las convenciones comerciales de los textos más demandados socialmente; b) evaluación crítica de los factores lingüísticos y culturales que condicionan el discurso comercial; c) aplicación de las estrategias traslativas según el encargo y tipo textual; d) conocimiento de los aspectos profesionales más importantes del ejercicio de la traducción comercial; e) manejo de las herramientas terminológicas y documentales más adecuadas y los recursos multimedia para la práctica de la traducción comercial.

A la vista de tales condiciones, el objetivo principal que nos planteamos en este trabajo es presentar una propuesta metodológica o un planteamiento formativo de inmersión temática que desentrañe la complejidad conceptual de los textos comerciales trabajados en el aula y que, al mismo tiempo, sirva de ayuda a los interesados en la enseñanza-aprendizaje de estos documentos especializados. Para ello, proponemos, en primer lugar, trabajar con la tipología textual del ámbito; en segundo término, establecer las convenciones textuales y dificultades más comunes de la traducción comercial y ofrecer posibles soluciones de acuerdo con el escopo de la traducción, y, en último lugar, con el fin de obtener una óptima preparación y formación de nuestros estudiantes en este ámbito de especialidad, desarrollar glosarios de términos que incluyan al menos los siguientes componentes: término en LO, término en 
LM, definición o explicación, tipo textual y fuentes utilizadas. En la siguiente sección, presentamos las opiniones obtenidas a partir de encuestas realizadas a los estudiantes que han cursado la materia en los grados de la Facultad de Traducción e Interpretación (FTI) de la Universidad de Las Palmas de Gran Canaria (ULPGC) durante el curso 2014-2015 con el fin de conocer su valoración sobre aspectos que nos parecían relevantes con respecto al planteamiento formativo recibido. Nuestro trabajo se cierra con unas conclusiones sobre los puntos más importantes abordados en él.

Ante todo, el aprendiz de traductor que se enfrenta por primera vez a la traducción comercial debe ser consciente de la complejidad y heterogeneidad de la materia, así como de la necesidad de adquirir un conocimiento especializado para el desarrollo de su competencia cognitiva y comunicativa. Recordemos que Rodríguez Camacho (2002: 312-313), entre otros investigadores, plantea las competencias del traductor de lenguajes especializados: cognitiva, que está centrada en el conocimiento del ámbito especializado; lingüística, que está relacionada con el dominio de dos o más lenguas; comunicativa, que es la capacidad para comunicar conocimiento especializado (escrito y oral), y metodológica, que es la capacidad para hacer el trabajo de forma ordenada y sistemática mediante la aplicación de un método y la utilización de herramientas y recursos documentales, terminológicos e informáticos disponibles.

Partiendo del convencimiento de que la competencia traductora en comercio internacional puede obtenerse con una metodología que contemple el desarrollo de las habilidades y conocimientos que requiere su enseñanzaaprendizaje, a continuación presentamos nuestra propuesta formativa para la enseñanza de esta materia, que pone énfasis en el proceso traductor.

\section{Propuesta formativa: algunas consideraciones}

Los beneficios de la explotación de géneros textuales tanto para la didáctica como para el ejercicio de la traducción y la comunicación especializada están más que consolidados, y prueba de ello son los diversos estudios, realizados en los últimos años, que han favorecido tanto la investigación como la docencia, y de los que algunos de los precursores son Swales (1990), Bathia (1993) o Trosborg (1997). En España, despuntan, entre otros, los trabajos que desarrolla el equipo de investigación Gentt (Montalt et al. 2008; Borja et al. 2012; García Izquierdo 2009; Gamero 2001), que se fundamentan en el concepto de género textual aplicado a los ámbitos de la comunicación especializada (jurídica, médica y técnica). Consideramos que el desarrollo de la 
competencia textual en la traducción comercial debe ser uno de los objetivos de la enseñanza de esta materia porque:

(...) las tareas de importación y exportación se van negociando fundamentalmente mediante la correspondencia comercial primero y, en una fase posterior, se materializan en los documentos mercantiles. Es gracias a estos [últimos] documentos como pueden finalmente llevarse a cabo operaciones comerciales con garantía de seguridad entre dos empresas, una compradora y otra vendedora, establecidas en países diferentes (Socorro Trujillo 2012: 304).

\subsection{Tipología textual}

Los textos comerciales son los que, directa o indirectamente, generan las distintas transacciones comerciales. Ciertos textos comerciales podrán tener carácter legal o jurídico cuando, fruto de ese texto, nazca una relación jurídica entre dos o más partes; una relación jurídica o contractual en el sentido de que surgen derechos y obligaciones entre dichas partes. Se trata de "textos estereotipados y en ocasiones repetitivos que constituyen géneros textuales perfectamente definidos ya que todos y cada uno de ellos tiene un objetivo comunicativo" (Borja 2007: 147). En efecto, como apunta Mayoral (2006: 6), "la uniformidad dentro de los tipos textuales es muy alta en el caso del comercio. La razón principal es que se ha producido un esfuerzo deliberado por normalizar el comercio internacional y, por ende, los documentos que genera". Estos serán objeto de traducciones reales en la vida profesional, ya que se tramitan con frecuencia en empresas involucradas en este ámbito de especialidad, como departamentos de exterior de bancos, sanidad exterior, compañías de seguros, consignatarias, puertos o empresas que se dedican a la importación y exportación de mercancías, administración comercial internacional, almacenamiento de productos, etc.

Puesto que el aprendiz de traductor carece de conocimiento especializado previo, necesita desarrollar esa competencia cognitiva a la que hemos aludido con anterioridad y de la que forma parte su "competencia textual" (García Izquierdo 2011). Se trata de conocer aspectos pragmáticos que resultan tan necesarios para su formación como los de carácter lingüístico. Para ello, aparte de las aportaciones teóricas de carácter general que el estudiante recibe por parte del docente sobre el campo de estudio, consideramos también prioritario contextualizar los textos originales que se trabajan en el aula con el fin de que el estudiante ubique el texto en su contexto, primero, y, después, conozca el propósito comunicativo textual. Así, en el aula trabajamos a partir de una taxonomía, flexible y dinámica, de textos comerciales o, 
dicho de otro modo, del sistema de géneros (terminología de Bazerman 1994: 82) del comercio internacional. Comenzamos a trabajar con textos propios de la correspondencia comercial o del género epistolar porque permiten: 1) introducir al alumnado en el campo de la especialidad, concretamente en los conceptos especializados y en las situaciones comunicativas de forma progresiva, pues es el instrumento de comunicación entre dos empresas, del que dependerá que un contacto o compra-venta se impulse o se frustre, y 2) presentar y contextualizar los documentos mercantiles que se mencionen por esta vía y con los que trabajamos, en una fase posterior, de forma general, primero, e individual, después, pues es en ellos donde se materializa la operación comercial. Los textos del género epistolar pueden clasificarse de acuerdo con la función o intención textual (de reclamación, felicitación, petición de un pedido, etc.). Para abordar los documentos mercantiles, proponemos el trabajo con juegos de documentos porque, al ser los que se utilizan necesariamente en una transacción comercial, permiten estudiar tipos textuales distintos y representativos del comercio internacional propios de una situación comunicativa concreta. La taxonomía de documentos mercantiles en la que nos apoyamos para completar la competencia textual del alumnado es la de Socorro Trujillo (2008: 45-124), quien los agrupa y clasifica de tal manera que resulta útil y sencillo localizarlos y contextualizarlos antes de trabajarlos individualmente. Dicha taxonomía, que a su vez abarca géneros y subgéneros, que no aportamos aquí por no ser el objetivo del presente trabajo, incluye los cuatro macro-géneros siguientes: a) documentos informativos, que son los solicitados por las partes para la producción y las fases de la compraventa de las mercancías (factura comercial, lista de contenidos, etc.); b) documentos oficiales o administrativos, que son los relevantes para que las autoridades gubernamentales controlen los movimientos internacionales de mercancías (documentos aduaneros como los generados para mercancías comunitarias o para terceros países y para-aduaneros, como los sanitarios o los de calidad); c) documentos de transporte y acompañamiento, que incluyen los utilizados para servicios intermediarios, de transporte y de seguros (conocimiento de embarque, póliza de fletamento, certificado de seguro, etc.), y d) documentos financieros o los relacionados con los requisitos de los bancos para asegurar y controlar los pagos (las declaraciones de transacciones en el exterior entre residentes y no residentes, los medios de pago, etc.) .

Por otra parte, la tipología textual se convierte en un elemento organizador para el docente, pues los textos se seleccionan progresiva y ordenadamente de acuerdo con el grado de dificultad, es decir, atendiendo fundamentalmente a 
los criterios de complejidad textual (PISA 2007: 28) y densidad terminológica textual (cfr. Cabré 1999).

Una vez el estudiante ha contextualizado cada tipo textual, deberá definir el propósito comunicativo porque, de esta manera, entenderá mejor la situación de comunicación y el uso social de los textos. A continuación, proponemos un análisis lingüístico por tipo textual, teniendo en cuenta las particularidades de los documentos a nivel general, primero, e individual, después. No nos detenemos a precisar el estudio textual del género epistolar aquí porque ya lo hemos abordado en otra aportación (Socorro Trujillo 2012: 307-320). Cabe añadir que esta forma de trabajar puede aplicarse a cualquier tipo textual de uso habitual que generen empresas relacionadas con el comercio y cuyas traducciones sean factibles (en Canarias, por ejemplo, hay una presencia importante de consignatarias y plataformas petrolíferas que producen un importante volumen de textos).

\subsection{Convenciones textuales de los documentos mercantiles: problemas, dificultades y soluciones}

\subsubsection{La intertextualidad y otros aspectos textuales}

La competencia textual del traductor especializado implica no solo un conocimiento de los géneros textuales del ámbito sino "un conocimiento adecuado de los esquemas conceptuales que vertebran el TO, de la estructura retórica de los tipos textuales concretos de un mismo género, así como de las normas que gobiernan la producción de textos [...] en la comunidad lingüístico-cultural de origen y llegada" (Corpas 2004: 139-140). Cabe señalar que los textos comerciales se caracterizan por la presencia de elementos no verbales (sellos, firmas, logos, membretes...) y verbales (de carácter lingüístico). El documento mercantil, concretamente, es un texto de naturaleza híbrida que presenta una parte, normalmente en el anverso, con un estilo formulario (con lenguaje bloque debido a la falta de espacio, esto es: con simplificación de estructuras oracionales, abundante nominalización, abreviaturas o siglas en muchos casos normalizadas internacionalmente, palabras truncadas, ausencia de pronombres personales e intensificadores, etc.). Esta parte del documento que, por regla general, se corresponde con la primera página o la cara frontal del documento, es la parte abreviada (short form) y variable del documento, porque es donde se insertan los datos relativos a una transacción comercial concreta. Por otro lado, está la parte invariable del documento (long form), que es aquella en la que se inserta la normativa o la jurisdicción que se aplica al documento en cuestión, a menudo en forma de cláusulas y, por tanto, la que presenta unas características 
propias del lenguaje jurídico. Este último tipo de lenguaje suele incluirse en algún espacio de la primera cara del documento, en el caso de los documentos más breves, como las facturas o algunos certificados de origen, o al dorso o reverso, en otras muestras como los certificados de seguro o los documentos de transporte marítimo. Por ello, nos atrevemos a afirmar que la propia naturaleza de los documentos mercantiles hace que la intertextualidad (Kristeva 1969), que plantea la relación directa de un texto con uno o varios textos anteriores, sea una característica manifiesta en estos tipos textuales. Aparte de estas dos variedades de lenguaje prototípicas de los documentos mercantiles, también, en la parte del documento correspondiente a la descripción de las mercancías, dependiendo de su naturaleza, los textos pueden contener tecnicismos que nada tienen que ver con el lenguaje legal o comercial, lo que corrobora la naturaleza híbrida de estos tipos textuales. Por tanto, la intertextualidad es un aspecto con el que el futuro traductor debe familiarizarse, ya que no solo debe discernir los distintos marcadores textuales de cada parte del TO, sino adaptarlos de forma adecuada a la hora de producir un TM.

\subsubsection{La normalización y el encargo de traducción}

Cabré (2002: 8) define "normalización" como:
a) el proceso de convertir o convertirse en norma, entendida como patrón, estándar o forma de referencia. En este sentido, se utiliza también el término "estandarización" y;
b) el proceso de convertir o convertirse en normal, es decir, en usual o habi- tual, el proceso cuyo objetivo es la extensión del uso de una lengua.

En efecto, al estudiar los textos comerciales, hemos podido constatar el esfuerzo que se hace en el comercio internacional por normalizar los documentos que se generan. La Conferencia Marítima Internacional del Báltico (BIMCO), por ejemplo, publica unos modelos de conocimientos de embarque y pólizas de fletamento, aunque no son los únicos, estandarizados internacionalmente. Además, existen frases, códigos y abreviaturas -como la norma ISO 4217 de las divisas del mundo, el código UNE-EN o ISO 445:2013 que regula las medidas y tipos de paletas o pálets para la manipulación de mercancías- normalizados internacionalmente, a los que podemos denominar "internacionalismos", según terminología de Gómez Capuz (2005: 57). ${ }^{1}$ Todos ellos facilitan el acceso a nuevos mercados, el comercio internacional y el tráfico de mercancías, y son establecidos por organismos distintos, como la Organización Internacional para la Normalización (ISO), la Organización

1. Este autor los incluye dentro de las categorías especiales o marginales del préstamo. 
Marítima Internacional (OMI), la Cámara de Comercio Internacional (CCI), Lloyd's y el Institute of London Underwriters (ILU) o la Organización Mundial del Comercio (OMC). La ISO, por ejemplo, es una entidad internacional encargada de favorecer las normas de fabricación, el comercio y la comunicación en todo el mundo. Con sede en Ginebra, es una federación de organismos nacionales entre los que se incluyen AENOR en España, DIN en Alemania o AFNOR en Francia. Su efectividad es incuestionable. Por supuesto, no existen normas para cada producto o servicio, y gran parte del comercio se lleva a cabo, en la práctica, directamente entre empresas por medio de contratos privados que se mantienen fuera del alcance del sistema de comercio multilateral. Este hecho representa un reto constante para la normalización.

La incorporación y uso de las normas técnicas apropiadas en la industria, por ejemplo, genera ventajas competitivas y comparativas al garantizar la idoneidad de un producto, el cual, al estar normalizado -y en el mejor de los casos- certificado con un sello de calidad, tiene el garante de ser parte de un modelo controlado, que unifica, tecnifica su forma de producción y considera aspectos vitales para el consumidor o usuario, lo que de paso representa valores agregados importantes a los ojos del mercado. Por ello, podemos afirmar que ciertas normas, como las ISO o los Incoterms de la Cámara de Comercio Internacional (CCI), se han convertido en un requisito importante para ingresar y operar en los mercados internacionales, y su aplicación incide positivamente en el posicionamiento y la competitividad de las empresas, sus productos y servicios, por lo que contribuyen asimismo a su penetración comercial. La propia ISO, en su página www.iso.org, se refiere en los siguientes términos a los beneficios de la estandarización para el ámbito de los negocios:

[...] [T] hey are are strategic tools that reduce costs by minimizing waste and errors, and increasing productivity. They help companies to access new markets, level the playing field for developing countries and facilitate free and fair global trade.

International Standards bring technological, economic and societal benefits. They help to harmonize technical specifications of products and services making industry more efficient and breaking down barriers to international trade. Conformity to International Standards helps reassure consumers that products are safe, efficient and good for the environment.

Por todo lo anteriormente expuesto, nos planteamos que el futuro traductor debe ser capaz de identificar estas frases, códigos y expresiones normalizadas a nivel internacional, y también debe ser capaz de estudiar y determinar las estrategias traslativas más adecuadas (según el encargo y el tipo textual). Es cierto que el uso habitual de unas formas estandarizadas facilita la interacción entre especialistas de un mismo campo, de manera que puedan comunicarse 
haciendo uso de esta normalización aunque no posean una competencia comunicativa en la lengua de intercambio. Esto, desde el punto de vista de la traducción, significa que entre especialistas no parece necesario traducir ni explicar muchos términos normalizados internacionalmente porque los conocen, caso en el que estos datos se transfieren a la hora de producir un TM. Precisamente, Newmark (1988) define "transferencia" como el procedimiento de traducción que consiste en copiar una palabra de un TO a un TM sin modificarla o en mantener el término intacto. Jaaskelainen (1999: 71), por su parte, define "estrategia" de la manera siguiente:

A series of competencies, a set of steps or processes that favor the acquisition, storage, and/or utilization of information. Strategies are heuristic and flexible in nature, and their adoption implies a decision influenced by amendments in the translator's objectives.

Sin embargo, como contempla la escuela funcionalista alemana, la finalidad de uso de un texto meta no tiene por qué ser necesariamente idéntica a la del texto original (Reiss \& Vermeer 1996). Es posible que las traducciones no siempre vayan destinadas a especialistas o a expertos en una materia. Por ejemplo, una traducción puede ser objeto de un juicio o un pleito legal, donde intervienen tanto noveles como expertos en la materia; o bien puede haber sido solicitada por un cliente que desee iniciarse en el mundo del comercio; o, por el contrario, tener una función fundamentalmente informativa dentro del ámbito de una empresa cuya correcta interpretación resulte vital para una operación comercial o financiera. Por lo tanto, la estrategia de traducción que se aplique tampoco tiene por qué ser la misma en todos los casos. En conclusión, el aprendiz de traductor debe ser consciente de este hecho, así como de saber qué estrategia de traducción aplicar (si la intraducibilidad o transferencia, la traducción o, en su caso, la ampliación de información) para evitar la infra-traducción o las pérdidas semánticas (Corpas Pastor 2001: 70) o lo directamente opuesto: la sobre-traducción o la adición de información innecesaria. En cualquier caso, el encargo de traducción determinará cuál es la estrategia más adecuada y el docente debe "guiar" al alumnado en este sentido.

\subsubsection{La terminología comercial: propuesta de elaboración de glosarios terminológicos}

La traducción comercial, como la traducción de cualquier ámbito de especialidad, constituye la finalidad de comunicar entre expertos de distintas lenguas. Por tanto, es indiscutible la necesidad que esta tiene de la terminología para expresar el conocimiento especializado de forma adecuada, conocimiento con 
el que el traductor actúa de mediador asumiendo las mismas competencias que el especialista. De hecho, Cabré (2000: 6) afirma:

Si las unidades terminológicas son el modo privilegiado de expresión del conocimiento, podemos decir que su primera función es representar dicho conocimiento. Cada unidad terminológica corresponde a un nudo cognitivo dentro de un campo de especialidad y el conjunto de dichos nudos conectados por relaciones específicas constituye la representación conceptual de dicha especialidad. Si ello es así, no cabe duda de que mediante la terminología representamos la realidad especializada.

"Traducir presupone conocer la materia que se traduce (conocer y comprender los términos) y saber expresarla con precisión y adecuadamente como lo haría un especialista" (Cabré 1999: 188). Por otra parte, en los últimos años, el mundo de la traducción ha experimentado una importante transformación tanto en lo que se refiere a los textos que se traducen como al uso de las herramientas informáticas aplicadas a la traducción (véanse, por ejemplo, los estudios al respecto de Austermühl 2001; Alcina 2002; Bowker 2002), que se utilizan en prácticamente todas las fases de la traducción: desde el momento en el que se crea el documento original, durante el proceso propiamente dicho de traducción o de elaboración y búsqueda de recursos de ayuda (glosarios, bases de datos terminológicos, corpus lingüísticos o textos paralelos, etc.), hasta el momento en el que se le da el formato final a la traducción.

Cabe recordar que los textos comerciales presentan un formato particular con estilo formulario, al menos en su cara frontal, que resulta más efectivo imitar en las traducciones. De acuerdo con Mayoral (2013: 206), aunque con referencia al formato de la traducción jurada de documentos de registro civil, optamos por el segundo sistema:

Caben dos sistemas extremos: el más antiguo o tradicional, que consiste en conservar el texto del documento impreso en una serie de párrafos consecutivos que siguen el orden de lectura normal en nuestra cultura o; el más moderno y facilitado por las aplicaciones informáticas, que consiste en replicar el formato original.

Por estas razones, consideramos que, en la medida de lo posible, el estudiantado de esta materia debe disponer de ordenador tanto dentro como fuera del aula, para la reproducción del documento original -que en ocasiones el traductor depura y mejora cuando contiene elementos ilegibles por el fotocopiado, formas abreviadas o errores gramaticales o de otra naturaleza (como, por ejemplo, la diglosia)-, ${ }^{2}$ la búsqueda de información y el desarrollo de

2. Entendida como "la alternancia de uso de un idioma oficial y otro alternativo"; no es extraño encontrar textos donde el usuario que los cumplimenta combina sus lenguas 
glosarios ya que, aunque el alumno no siempre necesite interiorizar todos los conocimientos especializados, sí precisa consultarlos y comprenderlos para poder resolver correctamente sus traducciones. No obstante, aunque el proceso de obtención de esa información podrá variar considerablemente en función de las competencias tecnológicas (dominio de herramientas TAO), los conocimientos lingüísticos y temáticos del estudiante, así como del nivel de especialización del documento que se pretenda traducir, en general, en traducción comercial, el trabajo con los recursos lexicográficos de uso habitual (diccionarios con o sin definiciones monolingües, bilingües o multilingües, textos paralelos, etc.) permite al estudiante localizar información sobre la materia para suplir sus carencias cognitivas. Además, cuanto mayor sea su conocimiento, mayor será su nivel de especialización. Para potenciarlo en el alumnado, proponemos la elaboración de glosarios terminológicos. Contreras (2014: 1) lo corrobora:

No se puede traducir sin comprender íntegramente el texto original, no se puede traducir sin cultura y tampoco se puede traducir sin retener. Aunque dispongamos de excelentes herramientas asistidas por ordenador, no dejan de ser instrumentos de ayuda; una memoria de traducción no recuerda por ti, sino que almacena datos para su uso posterior. Así pues, el mejor disco duro sigue siendo nuestro cerebro: cuanto más y mejor aprendamos, más y mejor comprenderemos y retendremos, y la retención en el ámbito de la traducción y profesiones afines resulta fundamental, especialmente en términos de rentabilidad. Nuestra memoria semántica es nuestro diccionario mental, y debemos procurar mantenerla lo más activa posible con una preparación apropiada basada en el esfuerzo añadido y en la asociación mental de ideas. Elaborar un glosario terminológico y fraseológico supone tiempo, dedicación y laboriosidad, aspectos nada desdeñables si pretendemos ayudar a nuestra memoria.

Por lo tanto, insistimos en que el estudiante ha de desarrollar glosarios de términos de cada tipo textual por varios motivos: 1) porque favorece la adquisición de conocimiento especializado y la competencia textual, 2) porque la polisemia es una dificultad a la que se enfrenta el aprendiz de traductor ya que hay términos que pueden traducirse de forma distinta en función del tipo textual en el que aparezcan -tal es el caso de draft (será el "calado" de un barco en documentos de transporte marítimo o un "efecto de comercio" o "letra" en los documentos de pago)-, y 3) porque el estudiante adquiere buenos hábitos de trabajo al tiempo que genera un recurso que puede ser de utilidad tanto

de trabajo de forma espontánea e incluso con errores gramaticales. [Ejemplo obtenido de un conocimiento de embarque marítimo: " 5 contenedores conteniendo 4076 cartons rones CIF landed Las Palmas"]. 
para su etapa de formación como para el ejercicio de su profesión, caso en el que podría ampliar sus competencias. Además, al elaborar glosarios bilingües, el estudiante cubre la dimensión lingüística, la cognitiva y la traslativa, todas ellas necesarias para la traducción comercial. De esta manera, se establece un vínculo entre la traducción pedagógica y la profesional puesto que con un planteamiento formativo de expertos en traducción comercial se plantea un método de traducción susceptible de servirle al estudiante en su futuro profesional. Pero, como ya hemos indicado, proponemos que se incluyan al menos los siguientes campos por término: término en LO, término en LM, definición o explicación, tipo textual y fuentes utilizadas. Con estos campos se cubren las necesidades mínimas del aprendiz de traductor. A continuación, mostramos ejemplos de dos entradas terminológicas en las que se recogen los campos mencionados con respecto a los términos CHOPT y option:

(1) Primer ejemplo

CHOPT (Charterer's Option) (Español) CHOPT (a opción del fletador) (Definición) A opción del fletador (véase option). (Tipo textual) Póliza de fletamento. (Fuente) CEMA (2000). Curso de terminología marítima. Centro de Estudios Marítimos del Atlántico, Las Palmas de Gran Canaria.; Acronym Finder Versión electrónica: <http://www.rimship.no/sider/liste.html>

(2) Segundo ejemplo

option (Español) opción (Definición) 1) Acuerdo por el que se establece el derecho a comprar (o a vender) un determinado bien, a un precio y dentro de un período preestablecidos. 2) Cláusula en Póliza de fletamento por la que se permite embarcar carga adicional o alternativa, "a opción del fletador", en puertos de escala a fin cargar o descargar una carga. 3) (Cláusula) Facultad que, en caso de siniestro, tiene la entidad aseguradora para efectuar la acción indemnizatoria eligiendo entre: a) abonar al que perjudica el importe en metálico de los bienes dañados; b) reparar los objetos perjudicados; c) reponer los bienes afectados por otros de análoga calidad. (Tipo textual) Póliza de fletamento. (Fuentes) Castelo Matrán, Julio \&Antonio Guardiola Lozano. (1992) Diccionario MAPFRE de Seguros, nueva edición trilingüe. Madrid: Fundación Mapfre; Branch, Alan. (1995) Dictionary of Shipping International Business Trade Terms and Abbreviations. London: Witherby \& Co. Ltd.

De esta manera, las labores de documentación (temática, terminológica, fraseológica, textual, etc.) del aprendiz de traductor quedan almacenadas.

\section{Los estudiantes opinan}

En este apartado presentamos los resultados de encuestas realizadas al alumnado de dos grupos diferentes de traducción comercial (un total de 69 estudiantes) de la Facultad de Traducción e Interpretación (FTI) de la Universidad de Las Palmas de Gran Canaria (ULPGC) durante el curso 2014-2015. Nos 
parece oportuno incluir las opiniones de los estudiantes en este trabajo porque nos ayuda a rectificar o a confirmar nuestras sospechas con respecto a algunas de las cuestiones abordadas con anterioridad en este trabajo y relacionadas con la docencia de la traducción comercial. Los estudiantes encuestados han cursado la asignatura de Traducción turístico-comercial inglés-español del grado de inglés-alemán y del grado de inglés-francés. La encuesta se ha efectuado concretamente al finalizar el bloque de traducción comercial (al que se dedica un total de 30 horas de docencia en cada grupo).

Habida cuenta de que los encuestados son estudiantes que han asistido regularmente a clase (69 presentes el día de la encuesta), se les formula un cuestionario de 12 ítems. Nueve preguntas de respuesta numérica, que valoran de 1 a 5 , puntuación donde 1 significa "Nada"; 2 , "Algo"; 3 , "Importante"; 4, "Muy importante", y 5, "Imprescindible". Y tres preguntas de respuesta abierta, a saber: justificación de la utilidad del glosario; en caso de que la respuesta sea afirmativa, se ha de especificar el tipo de fuentes más útiles para la traducción comercial, y opinar sobre la vinculación de la materia con el ámbito profesional. A continuación describimos brevemente los resultados de las encuestas.

Como vemos en el Gráfico 1, en el que se recogen las respuestas de las preguntas de respuesta numérica, todos los valores de las respuestas son positivos. Destaca, por ejemplo, que el $75,3 \%$ de los estudiantes (un total de 52) valore el glosario como "imprescindible" y que el $98,5 \%$ valore positivamente la utilidad del glosario ya que sus valores están entre el 3 y 5 . Además, en la pregunta de respuesta abierta en la que se les pide que justifiquen su respuesta, que no aparece en esta gráfica pero que hemos contabilizado, un $56 \%$ de los estudiantes responde que les parece útil porque "ayuda a asimilar conceptos" y el $45 \%$ opina que "facilita la traducción". Aunque se han recogido otras respuestas positivas, no las hemos considerado por ser diversas y no ser representativas; en otros casos, a pesar de que el estudiante ha hecho una valoración numérica positiva, no proporcionan una justificación, por lo que tampoco podemos aportar este dato. Con todo, sus respuestas corroboran nuestra idea de que, al elaborar sus glosarios con traducciones de los términos, definiciones y fuentes, estimulan la comprensión y su competencia tanto lingüística como cognitiva.

Otra cuestión que deseamos resaltar es la relacionada con "la necesidad de adquirir conocimiento especializado para la traducción comercial" y que se refleja en el último ítem de la gráfica. Al 7,2\% de los estudiantes no les parece necesario este conocimiento para afrontar la traducción comercial, frente al $92,7 \%$, que opina que sí. Cabe añadir que todos los estudiantes valoran por 
encima de 3 las siguientes cuestiones: conocer el significado de los términos tanto para la comprensión como la producción del TM, la adecuación de los tipos textuales trabajados en clase y la necesidad de trabajar con fuentes especializadas para la traducción comercial. En relación con la pregunta que versa sobre la vinculación de la asignatura con el ámbito profesional, la mayoría de las respuestas abiertas inciden en que supone una motivación el que a través de la materia puedan conocer la realidad socio-económica de su entorno y una posible salida profesional, así como el hecho de que se trabaje con documentos y encargos reales. Finalmente, aunque el dato general sigue siendo positivo, el 15,6\% manifiesta no interesarle este campo de especialidad.

En lo que se refiere a los tipos de fuentes consultadas que señalan los estudiantes, destacan los glosarios y sitios web especializados con definiciones, los textos paralelos y el material de clase. De todos estos apoyos, el 100\% de los alumnos consulta en algún momento, como es lógico, diccionarios y sitios web especializados con definiciones, el $20 \%$ recurre a textos paralelos, y el $42 \%$, al material de apoyo proporcionado en clase. En algunos casos consultan dos o más tipos de fuentes, incluidas las anteriores. Al tratarse de una pregunta de respuesta abierta, las respuestas no siempre son idénticas, aunque, en general, entre las fuentes más citadas destacan las siguientes: normas ISO (4217), guía de Incoterms, que localizan en sitios web de bancos o de cámaras de comercio, también webs de puertos o de empresas relacionadas con el comercio exterior, bases de datos terminológicas (la más consultada se localiza en iate.europa.

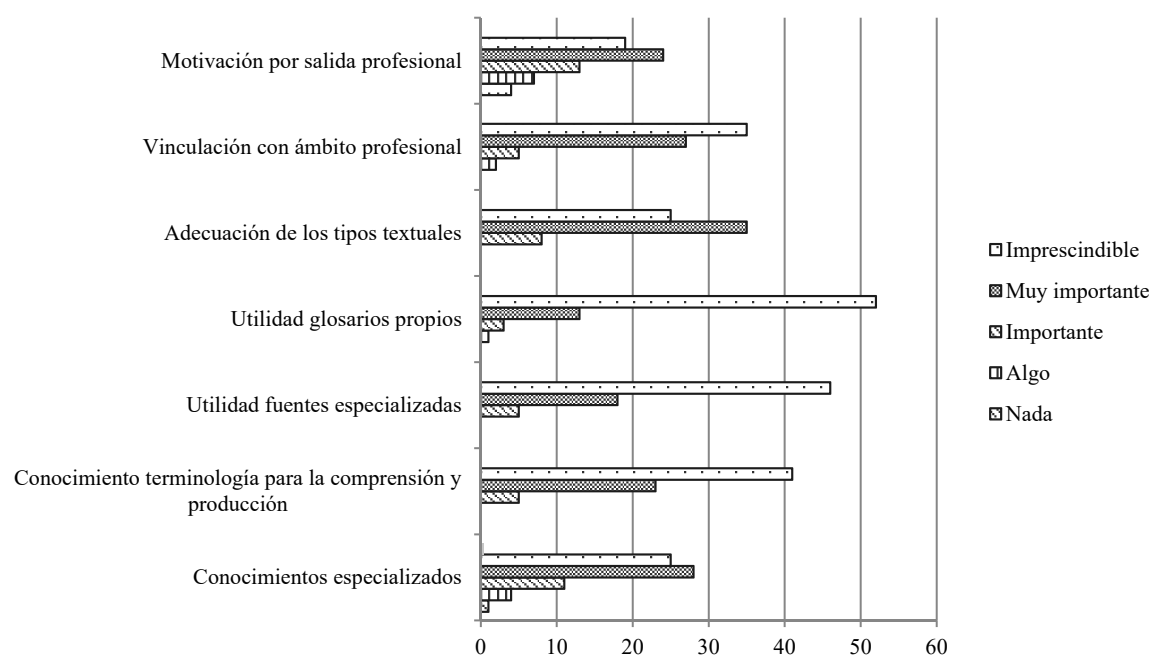

Gráfico 1. Valoración del alumnado sobre cuestiones relacionadas con la asignatura 
eu), Diccionarios de acrónimos y abreviaturas (Acronymfinder.com, Acronyms and abbreviations del Freedictionary.com), el glosario de comercio exterior de www.taric.es, Diccionario Comercio-exterior.es, International Trade Glossary del Translationjournal.net (Socorro Trujillo 2013), www.logisnet.com, Intercargo (glosario logístico), Andymiles.com, Diccionario de Mapfre, etc.

Finalmente, cabe señalar que, en las preguntas de respuesta abierta, se dan varios casos en los que los alumnos dejan las respuestas en blanco o sin cumplimentar a pesar de mostrarse positivos en las numéricas y, en otros, responden con varias respuestas distintas.

\section{Conclusiones}

Por regla general, el alumno-traductor que se enfrenta por primera vez a la traducción de los tipos textuales objeto de este estudio se encuentra con una materia compleja y heterogénea. Consideramos que la propuesta metodológica que aquí proponemos contempla la adquisición de las competencias que requiere la enseñanza-aprendizaje de esta materia, al enfocarse al desarrollo de la competencia cognitiva y comunicativa del estudiantado. En efecto, algunos problemas que plantea la traducción comercial son de carácter lingüístico, pero también de tipo cognitivo y pragmático (del tema, de los tipos textuales que se manejan, de los interlocutores y de la situación comunicativa). Además, contempla que los conceptos teóricos y el conocimiento especializado vayan ligados a la práctica. Es importante que el alumnado obtenga una base teórica y que domine los conceptos que se manejan en el comercio internacional antes de traducir. Debe comprender el TO o entender lo que contiene y ser capaz de redactar el TM conforme a las convenciones del TM en la CM (cultura meta) y su uso.

Asimismo, consideramos que el glosario elaborado por el estudiante -en el que recoge al menos los términos comerciales y sus equivalencias, los conceptos, el contexto y las fuentes- resulta un elemento útil del proceso traductor porque le permite ir asimilando los conceptos especializados al tiempo que lo familiariza con la materia y el ámbito. Por otra parte, también intentamos crear situaciones reales y modificar los encargos de traducción en la medida de lo posible mediante, por ejemplo, la incorporación de la modalidad de "traducción a la vista para clientes inexpertos". De este modo, se estimula el conocimiento especializado, ya que implica traducir con explicaciones y elaborar glosarios obligatoriamente. En definitiva, conviene recrear situaciones reales en las que el estudiante también necesite mostrar sus conocimientos. Aparte de esto, nos planteamos fomentar la intercambiabilidad de los glosarios entre los estudiantes. 
Un aspecto en el que necesitamos incidir es en la consulta de corpus lingüísticos como herramienta pedagógica en la enseñanza-aprendizaje de esta materia, así como en el uso de fuentes impresas por parte de los estudiantes ya que, a tenor de las respuestas de los encuestados, estos las emplean en una mínima proporción, prácticamente se conforman con el material de clase y son muy pocos los que consultan la bibliografía básica. Es obvio que resulta más fácil y cómodo conectarse a Internet. Un hándicap es el número elevado de alumnos, lo que imposibilita el acceso a la biblioteca. Una posible solución a este problema es la digitalización de estas fuentes bibliográficas impresas. Aparte de esto, hemos observado que, aunque no todos los estudiantes tienen las mismas preferencias, por regla general, sí parece motivarles lo mismo: el hecho de que se dé un enfoque profesional a la materia que se imparte. Por tanto, creemos que el docente, en la medida de lo posible, debe indagar sobre el mercado de su entorno laboral, los tipos textuales que más se generan, el tipo de encargos, e intentar simular situaciones reales. Asimismo, aunque supone una primera toma de contacto con la traducción comercial, conviene orientar a los estudiantes que deseen seguir formándose en este sector, en especial, sobre cursos de posgrado, maestrías, cursos de especialización, prácticas en empresas, becas, etc. Aunque nuestra metodología persiga, en principio, conseguir un conocimiento pasivo del tema que habilite al estudiante para comprender un TO y producir un TM adecuado, el egresado que se especialice en esta área experimentará una inmersión temática cada vez mayor y más activa, especialmente si acaba dedicándose a ello profesionalmente.

\section{Referencias bibliográficas}

Agencia Nacional de Evaluación de la Calidad y Acreditación. (2004) Libro blanco. Título de Grado en Traducción e Interpretación. Versión electrónica: <http://www.aneca.es/var/media/150288/libroblanco_traduc_def.pdf>

Alcina CAUdeT, Amparo (2002) "Estrategias y recursos de la informática aplicada a la traducción." En: Rodríguez Inés, Patricia (ed.) 2002. Papers de Tradumática. Actes del Primer Simposi sobrel'Ensenyament a distància i semipresencial de la Tradumàtica. Grup Tradumática, Universidad Autónoma de Barcelona (UAB). Versión electrónica: <http://www.fti.uab.cat/tradumatica/ papers/Papers_1.htm>

AUSTERMÜHL, Frank. (2001) Electronic Tools for Translators. Manchester: St. Jerome Publishing.

Bazerman, Charles. (1994) "Systems of Genres and the Enactment of Social Intentions." En: Freedman, Aviva \& Peter Medway (eds.) 1994. Genre and the New Rhetoric. London: Taylor and Francis, pp. 79-101. 
BHATIA, Vijay Kumar. (1993) Analysing Genre. Language use in professional settings. London \& New York: Longman.

Borja, Anabel. (2007) "Los géneros jurídicos." En: Alcaraz, Enrique et al. (eds.) 2007. Las lenguas profesionales y académicas. Barcelona-Alicante: ArielIULMA. Pp. 141-152.

BorJA, Anabel; Tomás Conde; Natividad Juste \& Pilar Ordóñez. (2012) "A Genrebased Approach to the Teaching of Legal and Business English: the GENTT Specialized Corpus in the LSP Classroom." En: Bárcena, Elena; Timothy Read \& Jorge Arús (eds.) 2012. Selected Papers of the TISLID'10 Conference. London: Springer-Verlag.

BOWKer, Lynne. (2002) Computer-Aided Translation Technology. A Practical Introduction. Ottawa: Presses de l'Université d'Ottawa.

CABRÉ, María Teresa. (1999) La terminología: representación y comunicación. Barcelona: Institut Universitari de Lingüística Aplicada, Universitat Pompeu Fabra.

CABRÉ, María Teresa. (2000) "La enseñanza de la terminología en España: problemas y propuestas." Hermeneus 2, pp. 41-44.

CABRÉ, María Teresa. (2002) "Terminología y normalización lingüística”. Versión electrónica: <http://www.ehu.eus/documents/2430735/2877801/cabret.pdf>

CABRÉ, María Teresa. (2006) La Terminología: representación y comunicación: Elementos para una teoría de base comunicativa y otros artículos. Barcelona: Institut Universitari de Lingüística Aplicada (IULA), Universitat Pompeu Fabra (UPF).

CONTRERAS BlANCO, Fernando. (2014) “¿Interrelación cultural o retención terminológica?” La Linterna del Traductor 10. Versión electrónica: <http://www. lalinternadeltraductor.org/n10/interrelaciones-culturales.html>

CORPAS PASTOR, Gloria. (2001) "La creatividad fraseológica: efectos semánticospragmáticos y estrategias de traducción." Paremia 10, pp. 67-78. Versión electrónica: <http://www.paremia.org/wp-content/uploads/P10-8.pdf>

CORPAS PASTOR, Gloria. (2004) "La traducción de textos médicos especializados a través de recursos electrónicos y corpus virtuales." En: González, Luis \& Pollux Henúñez (eds.) 2004. Palabras de traductor, Actas del II Congreso El español, lengua de traducción. Bruselas, ESLETRA. pp. 137-164. Versión electrónica: <http://cvc.cervantes.es/lengua/esletra/pdf/02/017_corpas.pdf>

Del Pozo Triviño, Isabel. (2009) "La traducción de documentos marítimos. Clasificación de los principales géneros y marco de análisis." Sendebar 2, pp. $1-39$.

Del Pozo Triviño, Isabel. (2014) "Analysis of Charterparty Agreements from Textual Genre and Translation Points of View.” Meta 59:1, pp. 160-175. 
FONDO MONETARIO INTERNACIONAL. (FMI) (2001) "La liberalización del comercio mundial y los países en desarrollo". Versión electrónica: <https://www. imf.org/external/np/exr/ib/2001/esl/110801s.htm>

Gallego Hernández, Daniel. (2013) "Proyecto COMENEGO: algo más que un corpus multilingüe de economía y negocios.” En: Tortosa Ybáñez, Ma Teresa; José Daniel Álvarez Teruel \& Neus Pellín Buades (coords.) 2013. X Jornadas de Redes de Investigación en Docencia Universitaria. La participación y el compromisode la comunidad universitaria. Alicante: Editorial ICE/Vicerrectorado de Estudios e Innovación Educativa, Universidad de Alicante, pp. 2242-2251.

Gallego Hernández, Daniel \& Ramesh Krishnamurthy. (2013) "COMENEGO (Corpus Multilingue de Economia y Negocios): design, creation and applications." Empirical Language Research 7. Versión electrónica: <http://ejournals. org.uk/ELR/article/2013/1>

GAMERO PÉREZ, Silvia. (2001) La traducción de textos técnicos: Análisis de géneros. Barcelona: Ariel.

GARCía IZQUIERDO, Isabel. (2009) Divulgación médica y traducción. El género Información para pacientes. Bern: Peter Lang.

GARCÍA IZQUIERDO, Isabel. (2011) Competencia textual para la traducción. Valencia: Tirant lo Blanch.

GÓmEZ CAPUZ, Juan. (2005) La inmigración léxica. Madrid: Arco/Libros.

JAASKELAINEN, Riitta. (1999) Tapping the process: an explorative study of cognitive and effective factors involved in translating. Joensuu: University of Joensuu Publications in Humanities.

KrISTEVA, Julia. (1969) Recherches pour une sémanalyse. Paris: Éditions du Seuil.

MAYORAl ASENSIO, Roberto. (2006) "La traducción comercial." Butlletí de la Associació de Traductors i Interprets Jurats (diciembre). Versión electrónica: <http://www.ugr.es/ rasensio/docs/Traduccion_comercial.pdf>

MAYORAL ASENSIO, Roberto. (2007) "La traducción comercial." En: Fuertes, Pedro (ed.) Problemas lingüísticos en la traducción especializada. Valladolid: Secretariado de Publicaciones, Universidad de Valladolid, pp. 33-47.

Mayoral AsEnsio, Roberto. (2013) "Guía para la traducción jurada de documentos de registro civil (nacimiento y defunción) del inglés al español." Panacea XIII:36, pp. 202-228. Versión electrónica: <http://www.tremedica.org/panacea/IndiceGeneral/n36-tradyterm_RMayoralAsensio.pdf>

NeWmark, Peter. (1988) A Textbook of Translation. Londres: Prentice Hall.

Medina Reguera, Ana María. (2007) "La Traducción Alemán-Español de Documentos del Comercio Exterior: Organización de la Tipología e integración del contenido temático." En: Santana, Belén; Silvia Roiss \& $\mathrm{M}^{\mathrm{a}}$ Angeles Recio (Eds.) Puente Entre Dos Mundos: Últimas Tendencias en la Investigación Traductológica Alemán-Español. Salamanca: Universidad de Salamanca, pp. 252-260. 
Medina Reguera, Ana María. (2009) “Análisis de Necesidades en la Programación de Cursos en Contextos Profesionales: Estadística Sobre la Enseñanza de Español de los Negocios en España.” En: Fernández, Inmaculada \& Agustín Vera Luján (eds.) 2009. El Español en Contextos Específicos: Enseñanza e Investigación. Fundación Comillas, pp. 763-780.

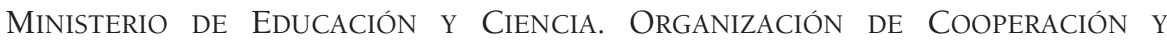
Desarrollo ECONÓmico (OCDE). PISA (2007) "La lectura en PISA (2000, 2003 y 2006). Marco y pruebas de evaluación”. Madrid. Versión electrónica: <http://www.mecd.gob.es/dctm/ievaluacion/internacional/pisalectura.pdf?do cumentId=0901e72b8010c470>

Montalt Resurreció, Vicent; Pilar Ezpeleta Piorno \& Isabel García Izquierdo. (2008) "The Acquisition of Translation Competence through Textual Genre." Translation Journal 12:4, pp. 1-12.

REISS, Katharina \& Hans Vermeer. (1996) Fundamentos para una teoría funcional de la traducción. Madrid: Akal Universitaria.

RodríGuez-InÉs, Patricia. (2014) "COMENEGO: Compilación del corpus piloto en inglés y primeros análisis.” En: Gallego Hernández, Daniel (ed.) 2014. Traducción económica: entre profesión, formación y recursos documentales. Los estudios de Traducción e Interpretación basados en corpus. I Coloquio Hermeneus. Soria: Diputación Provincial de Soria, pp. 187-199.

Rodríguez CAMACHO, Elena. (2002) "La terminología en la formación de un traductor especializado." En: Guerrero Ramos, Gloria \& Manuel Fernando Pérez Lagos (eds.) Panorama actual de la terminología. Granada: Comares, pp. 307-327.

RomÁn MíngueZ, Verónica. (2012) "Algunas peculiaridades de la traducción inglés-español del discurso de los negocios." En: Martino Alba, Pilar \& Christiane Lebsanft (eds) 2012. Telar de traducción especializada. Madrid: Dykinson, pp. 91-103.

Socorro Trujillo, Karina. (2008) Aspectos textuales y terminológicos de documentos mercantiles del comercio internacional. Servicio de publicaciones de la Universidad de Las Palmas de Gran Canaria (ULPGC).

Socorro Trujillo, Karina. (2012) "La enseñanza de la traducción directa de la correspondencia comercial: hacia una competencia textual del comercio internacional." Sendebar 23, pp. 301-320.

SOCORRO TRUjILlo, Karina. (2013) "International trade glossary." Translation Journal 7:1. Versión electrónica: <http://translationjournal.net/ journal/63trade.htm>

SUAU JiMÉNEZ, Francisca. (2010) La traducción especializada en inglés y español en géneros de Economía y Empresa. Madrid: Editorial Arco/Libros.

SWALES, James. (1990) Genre analysis: English in academic and research settings. Cambridge: Cambridge University Press. 
Trosborg, Ana. (1997) "Text Typology: Register, Genre and Text Type." En: Trosborg, Ana (ed.) Text Typology and Translation. Amsterdam: John Benjamins, pp. 3-23.

\section{NOTA BIOGRÁFICA / BIONOTE}

KARINA SOCORRO, traductora, intérprete y filóloga, es profesora desde 1990 en la Facultad de Traducción e Interpretación (FTI) de la Universidad de Las Palmas de Gran Canaria (ULPGC). Su tesis doctoral (2002) versa sobre la traducción de los documentos mercantiles del comercio internacional y combina la docencia con el ejercicio profesional. Sus principales líneas de investigación son la traducción turística y comercial. Sobre esta última ha dirigido un proyecto de investigación y, en 2008, publicado el libro Aspectos textuales y terminológicos de documentos mercantiles del comercio internacional (prólogo de Roberto Mayoral Asensio). Ha pasado estancias de investigación (Dublin City University, Irlanda, Comillas...). Ha impartido seminarios y presentado ponencias en Irlanda (DCU o NUI de Galway), en Ginebra (UNIGE) o en Italia (la Sapienza), etc. Asimismo, tiene artículos y capítulos de libros publicados. Ha sido coordinadora del Máster de traducción (2010-2014) y miembro del comité científico de la revista de impacto de Lenguas para Fines Específicos de la ULPGC.

KARINA SOCORRO has a degree in Translation and Interpreting and in English philology. She has been a lecturer in Translation Studies since 1990 in the Faculty of Translation and Interpreting at the University of Las Palmas de Gran Canaria (ULPGC). Her PhD thesis (2002) focused on the translation of mercantile papers used in international trade. Her main lines of research are both tourist and commercial translation. With regards to the latter, she has directed a research project and has published the book: Aspectos textuales y terminológicos de documentos mercantiles del comercio internacional (prologue by Roberto Mayoral Asensio). She also combines translation teaching with professional translation. She has researched in DCU (Ireland), Comillas (Madrid), etc. She has given seminars and presented papers in Ireland (DCU or NUI Galway), Geneva (UNIGE), Italy (La Sapienza), etc. She has also published several articles and book chapters. And she has been the coordinator of the Master in translation studies and a member of the editorial committee of the journal Lenguas para Fines Especificos in the ULPGC. 